\title{
Numerical modelling of centrifuge dynamic tests of circular tunnels in dry sand
}

\author{
Riccardo Conti · Giulia M. B. Viggiani • \\ Francesco Perugini
}

Received: 3 April 2013/Accepted: 11 October 2013

(C) Springer-Verlag Berlin Heidelberg 2013

\begin{abstract}
This paper describes the numerical simulation of two dynamic centrifuge tests on reduced scale models of shallow tunnels in dry sand, carried out using both an advanced bounding surface plasticity constitutive soil model and a simple Mohr-Coulomb elastic-perfectly plastic model with embedded nonlinear and hysteretic behaviour. The predictive capabilities of the two constitutive models are assessed by comparing numerical predictions and experimental data in terms of accelerations at several positions in the model, and bending moment and hoop forces in the lining. Computed and recorded accelerations match well, and a quite good agreement is achieved also in terms of dynamic bending moments in the lining, while numerical and experimental values of the hoop force differ significantly with one another. The influence of the contact assumption between the tunnel and the soil is investigated by comparing the experimental data and the numerical results obtained with different interface conditions with the analytical solutions. The overall performance of the two models is very similar indicating that at least for dry sand, where shear-volumetric coupling is less relevant, even a simple model can provide an adequate representation of soil behaviour under dynamic conditions.
\end{abstract}

R. Conti ( $\bowtie)$

Università degli Studi Niccolò Cusano,

Via Don Carlo Gnocchi, 3, 00166 Roma, Italy

e-mail: riccardo.conti@unicusano.it; rconti@sissa.it

G. M. B. Viggiani · F. Perugini

DICII, Università di Roma Tor Vergata, Roma, Italy

e-mail: viggiani@uniroma2.it

F. Perugini

e-mail: francesco.perugini@live.it
Keywords Centrifuge test - Constitutive model · Earthquake - Interface friction - Numerical analyses . Tunnel

\section{Introduction}

The recent literature reports a number of case histories of damage to tunnels during earthquakes [17, 43], most of them related to racking and ovaling of the cross-section due to shear waves propagation [32]. These observations have led several researchers to investigate further the behaviour of underground structures under seismic actions, both numerically $[1,8,15,19,23,34,44]$, experimentally [9, $25,42]$, and with the analysis of specific case studies [12, 21], mainly to verify the closed-form solutions commonly adopted in the seismic design of tunnels.

Analytical solutions are generally developed for ovaling deformations of the transverse section of the tunnel, applying a quasi-static uniform strain field to the soiltunnel system and assuming linear elastic behaviour for both the soil and the lining [31, 36, 41]. Two limit cases are considered, in which either zero friction (full-slip condition) or perfect bond (no-slip condition) is assumed at the contact between the tunnel lining and the surrounding soil. As shown by Hashash et al. [17], significant discrepancies can be expected in the maximum internal forces computed using the different solutions available in the literature and, also, the assumption on the contact condition plays a major role in the computation of the hoop force acting in the lining.

Most numerical works presented in the literature have focused on the appropriate choice of the contact condition between the soil and the tunnel $[18,23,34]$ and on the 3D modelling of soil-structure interaction [19, 44] while 
paying less attention to the constitutive assumptions for the mechanical behaviour of the soil. As a matter of fact, Hashash et al. [18] and Sedarat et al. [34] used a linear elastic model for the soil, in order to reproduce the same conditions adopted in the closed-form solutions, while Kouretzis et al. [23] and Yu et al. [44] used a simple nonlinear hysteretic constitutive relation based on the wellknown Ramberg and Osgood [33] model.

A critical issue in the numerical simulation of dynamic soil-structure interaction phenomena is the choice of an adequate constitutive model for the soil [22]. A number of constitutive models have been proposed to reproduce the behaviour of non-cohesive soils under cyclic loading (see, e.g. Andrianopoulos et al. [2] and Zhang and Wang [45] for an extensive review). In principle, the constitutive model should permit to reproduce adequately at least: (1) the nonlinear and hysteretic behaviour of soil with increasing deformation, which plays a crucial role in the amplification phenomena related to stress wave propagation, (2) the attainment of critical state conditions at large deviatoric strains and (3) the static and dynamic liquefaction related to excess pore pressure build-up in undrained loading. Ideally, the model should use a single set of parameters, calibrated from the results of standard laboratory tests.

The work described in this paper originated from an invitation to participate to a round-robin numerical test on the behaviour of tunnels under seismic loading (RRTT) launched jointly by TC104, TC203 and TC204 of the ISSMGE. The experimental results of one centrifuge test on a reduced scale model of a shallow tunnel in dense dry sand were made available to the scientific community in order to benchmark different numerical methods. At a later stage, the results of one further test on loose dry sand, recently presented by Lanzano et al. [25], were made available to extend the original exercise of blind numerical prediction.

In the work described in this paper, two different constitutive models were adopted for the soil, both implemented in the finite difference code FLAC [20]. These were an advanced constitutive model proposed by Andrianopoulos et al. [2,3] for non-cohesive soils (model M1) and a simple Mohr-Coulomb elastic-perfectly plastic model with embedded nonlinear and hysteretic behaviour (model M2).

The main objective of the work was to compare the predictive capabilities of the two constitutive models adopted for the soil, and to verify the influence of some numerical assumptions, such as the contact condition between the lining and the soil, on the internal forces in the lining. For this purpose, the paper presents an extensive comparison between experimental data, numerical predictions and analytical results.

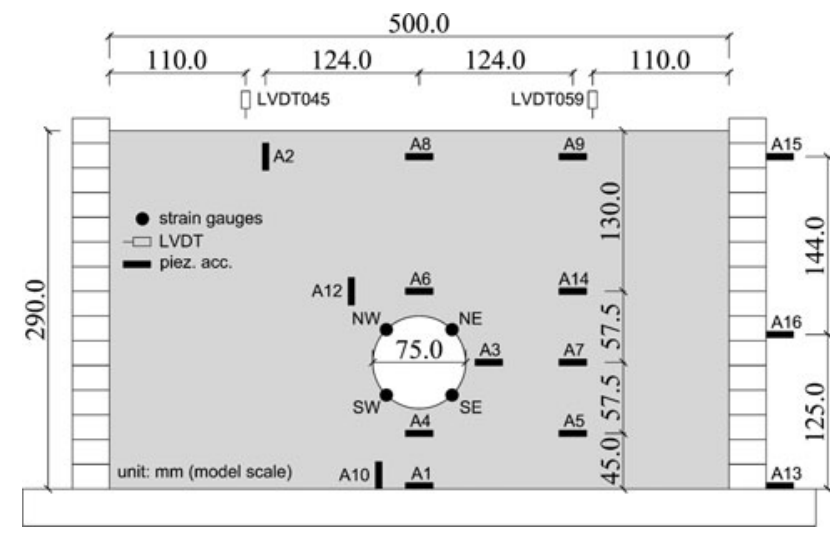

Fig. 1 Test T3 and T4: transducers layout

Table 1 Earthquake features (model scale)

\begin{tabular}{llllll}
\hline Test & \multicolumn{2}{l}{ Model T3 } & & \multicolumn{2}{l}{ Model T4 } \\
\cline { 2 - 3 } \cline { 5 - 6 } & $f(\mathrm{~Hz})$ & $a_{\max }(\mathrm{N} \mathrm{g})$ & & $f(\mathrm{~Hz})$ & $a_{\max }(\mathrm{N} \mathrm{g})$ \\
\hline EQ1 & 30 & 0.06 & 30 & 0.05 \\
EQ2 & 40 & 0.07 & & 40 & 0.07 \\
EQ3 & 50 & 0.10 & & 50 & 0.12 \\
EQ4 & 60 & 0.14 & 60 & 0.20 \\
\hline
\end{tabular}

\section{Centrifuge model tests}

Lanzano et al. [25] presented the results of four centrifuge dynamic tests on reduced scale models of shallow tunnels in dry sand, reconstituted at different values of relative density. In this paper, only the two experiments that were proposed for the RRTT are discussed, that is tests T3 $\left(D_{\mathrm{R}}=75 \%\right)$ and T4 $\left(D_{\mathrm{R}}=40 \%\right)$, both prepared within a laminar box container. Figure 1 shows the main geometrical quantities for the problem, together with the layout of instrumentation.

The tunnel lining was modelled using an aluminiumcopper alloy tube (density, $\rho=2,700 \mathrm{~kg} / \mathrm{m}^{3}$; Young modulus, $E_{1}=68.5 \mathrm{GPa}$; Poisson's ratio, $v_{1}=0.3$ ), with an external diameter $D=75 \mathrm{~mm}$ and thickness $t=0.5 \mathrm{~mm}$.

A standard fine silica sand, that is Leighton Buzzard (LB) sand, fraction $E, 100 / 170$, was used to prepare the models. The specific gravity of LB sand is $G_{\mathrm{S}}=2.65$, its maximum and minimum voids ratio are 1.014 and 0.613 , respectively, and its constant volume friction angle is $\phi_{\mathrm{cv}}=32^{\circ}$. A comprehensive characterisation of the mechanical behaviour of the sand has been presented by Visone [37] and Visone and Santucci de Magistris [38].

Instrumentation was used to measure accelerations at different locations in the model and on its boundaries, bending moments and hoop forces in the lining, and vertical displacements at the soil surface (see Fig. 1). 
Table 2 Main scale factors in geotechnical centrifuge modelling

\begin{tabular}{ll}
\hline Quantity & Scale factor \\
\hline Length & $1 / N$ \\
Time (dynamic) & $1 / N$ \\
Acceleration & $N$ \\
Stress & 1 \\
Strain & 1 \\
Force/unit length & $1 / N$ \\
\hline
\end{tabular}

During each test, the model was subjected to a series of five trains of approximately sinusoidal waves with different nominal frequencies, $f_{\text {inp}}$, and amplitudes, $a_{\max }$, and a constant duration of $0.4 \mathrm{~s}$ at model scale. The input accelerations were applied at the base of the models in the horizontal direction and recorded by accelerometer A13. Table 1 shows the main features of the first four earthquakes, applied at a centrifugal acceleration of $80 \mathrm{~g}$, which will be discussed in the present work.

In the following, accelerations are positive rightwards. All results are presented at model scale, unless explicitly stated. For sake of clarity, the main scale factors in geotechnical centrifuge modelling are reported in Table 2, where $N$ is the ratio between the centrifugal and gravitational acceleration.

\section{Constitutive models for the soil}

\subsection{Bounding surface plasticity (M1)}

Model M1 was developed by Andrianopoulos et al. [2, 3] within the framework of bounding surface plasticity and critical state soil mechanics, to simulate the mechanical behaviour of non-cohesive soils under small-to-large cyclic deformations. The main ingredients of the model, mostly derived from the original works by Manzari and Dafalias [27] and Papadimitriou et al. [30], are as follows: (1) the existence of three conical surfaces in the stress space (critical state, bounding and dilatancy), interrelated through the state parameter $\psi$ [4]; (2) kinematic hardening; (3) a nonlinear hysteretic formulation for the "elastic" moduli, which defines the shear modulus degradation and the hysteretic damping increase at small-medium shear strains; (4) a scalar multiplier for the plastic modulus, taking into account globally the sand fabric evolution during shearing. Note that, as the yield surface is not defined in the model, and hence no elastic domain exists, the terminology "elastic" used throughout the paper, and derived from Andrianopoulos et al. [2], refers simply to the behaviour of the soil at small strains.
Table 3 Model constants for the constitutive soil model M1

\begin{tabular}{|c|c|c|}
\hline Parameter & Physical meaning & Value \\
\hline$\Gamma$ & Void ratio at critical state $\left(p^{\prime}=1 \mathrm{kPa}\right)$ & 0.825 \\
\hline$\lambda$ & Slope of CSL in the $e-\ln p^{\prime}$ plane & 0.037 \\
\hline$M_{\mathrm{c}}$ & $\begin{array}{l}\text { Deviatoric stress ratio at critical state in } \\
\text { triaxial compression (TXC) }\end{array}$ & 1.346 \\
\hline$M_{\mathrm{e}}$ & $\begin{array}{l}\text { Deviatoric stress ratio at critical state in } \\
\text { triaxial extension (TXE) }\end{array}$ & 0.867 \\
\hline$k_{c}^{b}$ & $\begin{array}{l}\text { Effect of } \psi \text { on peak deviatoric stress ratio } \\
\text { (TXC) }\end{array}$ & 3.457 \\
\hline$k_{c}^{d}$ & $\begin{array}{l}\text { Effect of } \psi \text { on dilatancy deviatoric stress ratio } \\
\text { (TXC) }\end{array}$ & 1.041 \\
\hline$v$ & Poisson's ratio & 0.3 \\
\hline$B$ & Elastic shear modulus constant & $\begin{array}{l}800 \\
(600)\end{array}$ \\
\hline$a_{1}$ & Nonlinearity of elastic shear modulus & $\begin{array}{l}0.5 \\
(0.85)\end{array}$ \\
\hline$\gamma_{1}$ & $\begin{array}{l}\text { Reference shear strain for nonlinearity of } \\
\text { elastic shear modulus }\end{array}$ & 0.00025 \\
\hline$A_{0}$ & Dilatancy constant & 1 \\
\hline$h_{0}$ & Plastic modulus constant & 50,000 \\
\hline$N_{0}$ & Fabric evolution constant & 30,000 \\
\hline
\end{tabular}

The evolution equations defining the constitutive model are discussed in detail in many works (see, e.g. [2, 27, 29, 30]), and therefore they are not reported in this paper.

The constitutive model requires the definition of 13 constants, which can be calibrated from the interpretation of standard laboratory tests (see, e.g. [2, 30]). In this work, the model constants were calibrated using the experimental results presented by Visone and Santucci de Magistris [38], obtained with a variety of laboratory tests carried out on samples of LB Sand, reconstituted at different values of relative density. The sole constants defining the shear modulus degradation curve were calibrated against the centrifuge experimental data presented by Conti and Viggiani [11], as detailed in the following. Table 3 presents the complete set of values for the model constants adopted in this work. For sake of clarity, the constitutive equations used for the calibration of some constants are recalled in Fig. 2.

Constants $M_{\mathrm{c}}$ and $M_{\mathrm{e}}$ define the slopes of the critical state lines (CSL) in compression and extension in the triaxial plane of the stress invariants $q: p^{\prime}$, while $\Gamma$ and $\lambda$ define the CSL in the $e: \ln p^{\prime}$ plane. These constants were obtained from undrained triaxial extension tests (TX-EU), drained triaxial compression tests (TX-CD) and drained triaxial compression tests at constant mean effective stress (TX-CDp), where a critical state was attained (see Fig. 2a, b).

Constants $k_{c}^{b}$ and $k_{c}^{d}$, which relate the bounding and dilatancy surfaces to the critical state surface in the triaxial plane through the state parameter $\psi[4]$, were obtained 

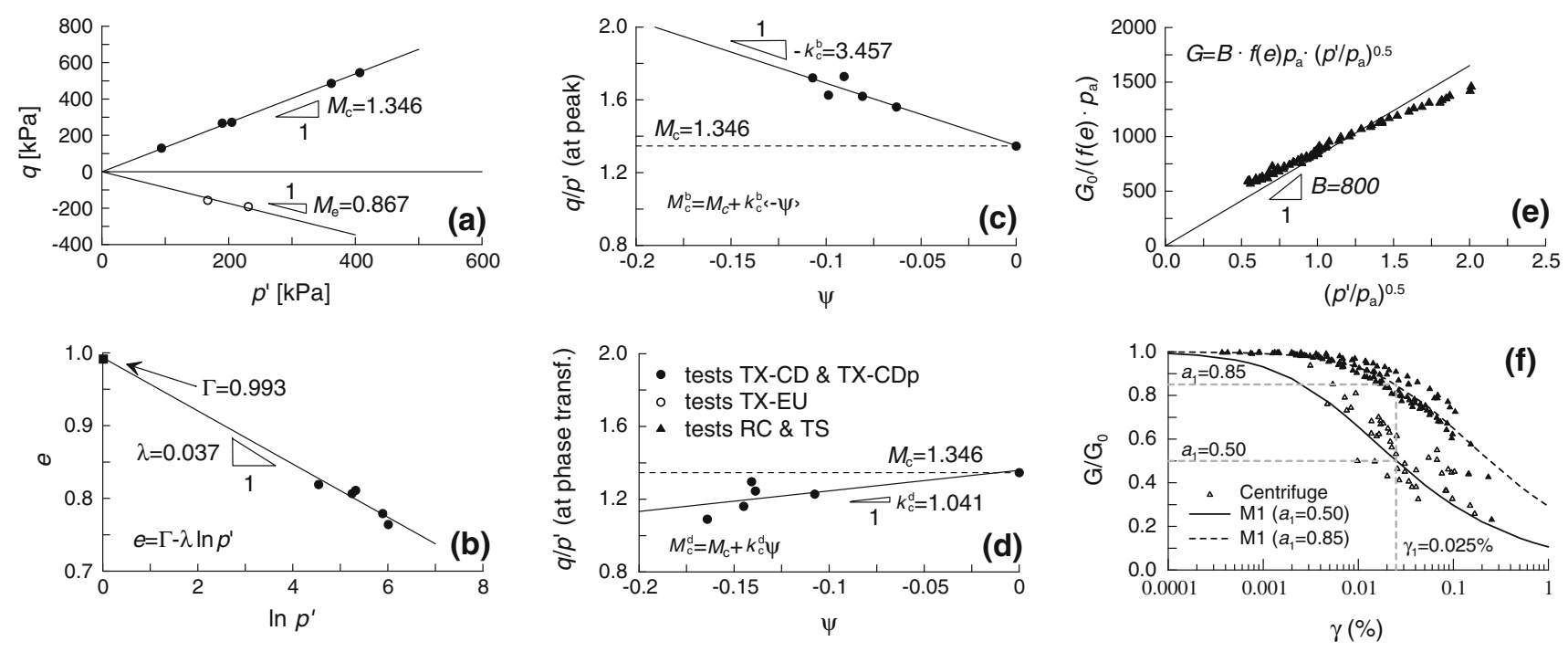

Fig. 2 Model M1: calibration of model constants from experimental data

from TX-CD and TX-CDp tests, by relating the deviatoric stress ratio $q / p^{\prime}$ at peak and at phase transformation, respectively, to the values of $\psi$ at which they are attained (see Fig. 2c, d).

Constant $B$, which defines the shear modulus at small strains, was estimated from resonant column (RC) tests carried out at different values of mean effective stress and voids ratio (see Fig. 2e). As observed by Papadimitriou et al. [30], values of $B$ obtained from small strain measurements are usually too large for accurate simulation of monotonic loading. Accordingly, a reduced value of $B$ (=600) was used for the numerical simulation of the static stage of the centrifuge tests, in plane strain (2D) analyses.

The constants $a_{1}$ and $\gamma_{1}$ define the shear modulus degradation curve: $\gamma_{1}(=0.025 \%)$ is related to the volumetric threshold shear strain, which ranges from $0.0065 \%$ to $0.025 \%$ for non-plastic soils [40], and $a_{1}$ is the corresponding value of $G / G_{0}$. Two different sets of experimental data were considered preliminary for the calibration of $a_{1}$ (Fig. 2f): (1) the laboratory (RC and TS) data reported by Visone and Santucci de Magistris [38], corresponding to which $a_{1}=0.85$, and (2) the centrifuge data presented by Conti and Viggiani [11], obtained from the interpretation of a number of centrifuge dynamic tests on model layers of LB Sand, corresponding to which $a_{1}=0.50$. The two sets of data are quite different, the latter showing a more rapid degradation of the shear modulus with increasing deformation, consistently with other literature data referring to LB Sand [7, 13] and non-plastic soils [35, 39]. As no convincing explanation could be found of the inconsistency between the two set of data, the value of $a_{1}=0.50$ was used in the $2 \mathrm{D}$ analyses, which provides a better match
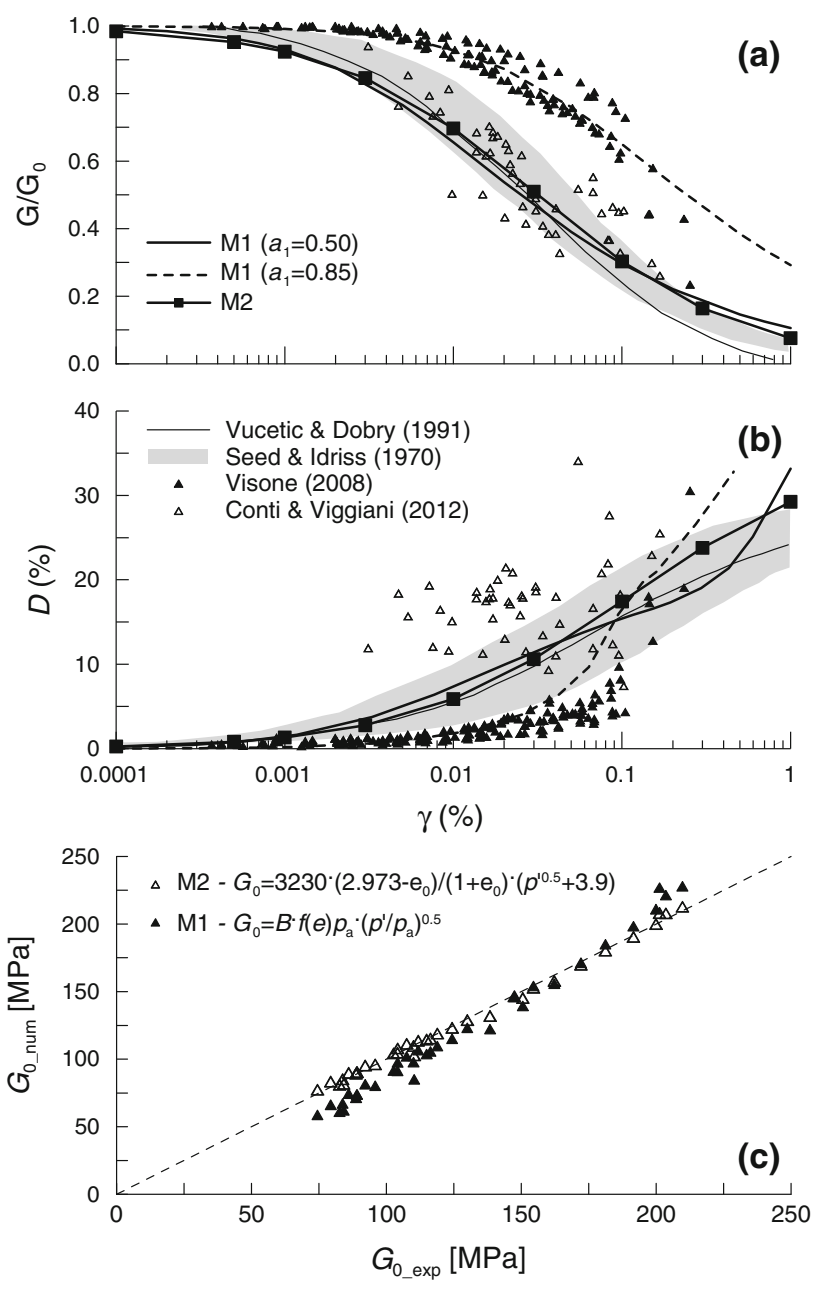

Fig. 3 Model M1 and M2. Calibration of model constants from laboratory and centrifuge data: a shear modulus degradation curve, b damping ratio and $\mathbf{c}$ small strain shear modulus 
between numerical and experimental accelerations within the soil layer. This is further discussed in the following section on the validation of the model.

The dilatancy constant, $A_{0}$, and the plastic modulus constant, $h_{0}$, were computed with a trial-and-error procedure, by fitting numerically the stress-strain response observed during TX-CD tests. Finally, in the absence of direct measurements, a value of 0.3 was used for the Poisson's ratio, $v$, while the value of the fabric constant, $N_{0}$, was chosen within the typical range provided by Andrianopoulos et al. [2].

\subsection{Perfect plasticity with embedded hysteretic behaviour (M2)}

Model M2 is a simple Mohr-Coulomb elastic-perfectly plastic model in which, during the dynamic stages, nonlinear and hysteretic behaviour is introduced for stress paths within the yield surface through a hysteretic model available in the library of FLAC 5.0 [20]. The hysteretic model consists in an extension to general strain conditions of the one-dimensional nonlinear models that make use of the Masing [28] rules to describe the unloading-reloading behaviour of soil during cyclic loading. Assuming that the stress state does not depend on the number of cycles, the relationship between shear stress, $\tau$, and shear strain, $\gamma$, can be written as:

$\tau=G_{\mathrm{S}}(\gamma) \cdot \gamma=G_{0} M_{\mathrm{S}}(\gamma) \cdot \gamma$

where $G_{\mathbf{S}}(\gamma)$ is the secant shear modulus, $G_{0}$ is the small strain shear modulus and $M_{\mathrm{S}}(\gamma)$ is the normalised secant shear modulus, defined as:

$M_{\mathrm{S}}=\frac{a}{1+\exp \left(-\left(\log _{10} \gamma-x_{0}\right) / b\right)}$

where $a, b$ and $x_{0}$ are model parameters that can be determined from the best-fit of a specific modulus degradation curve. Strain reversals during cyclic loading are detected by a change in the sign of the scalar product between the current strain increment and the direction of the strain path at the previous time instant. At each strain reversal, the Masing rule is invoked, and stress and strain axes are scaled by a factor of 0.5 , resulting in hysteresis loops in the stressstrain curves with associated energy dissipation.

The soil was modelled using a friction angle $\phi=32^{\circ}$, corresponding to the critical friction angle of LB Sand, and cohesion $c^{\prime}=0$, while a standard non-associated flow rule was adopted, with dilatancy angle $\psi=0$. The small strain shear modulus was computed using the expression proposed by Hardin and Drnevich [16]:

$G_{0}=3230 \frac{\left(2.973-e_{0}\right)^{2}}{1+e_{0}} \cdot\left(p^{\prime 0.5}+C\right)$ where $p^{\prime}$ is the mean effective stress, $e_{0}$ is the initial voids ratio of the sand and $C=3.9$ is a constant obtained from the best-fit of small strain RS tests on reconstituted samples of LB Sand [38]. Finally, soil parameters $a=1.0, b=-$ 0.6 and $x_{0}=-1.5$ were used for the normalised secant shear modulus in Eq. (2), derived from the best-fit of the modulus degradation curve obtained by Conti \& Viggiani [11]. Figure 3 shows a comparison between model predictions and laboratory data in terms of: (a) the modulus degradation curve, $G / G_{0}$, (b) the corresponding evolution of the damping ratio, $D$, with the mobilised shear strain, and (c) the small strain shear modulus. Figure $3 a, b$ also reports the upper and lower bound provided by Seed and Idriss [35] for dry sand (shaded area) and the experimental curve suggested by Vucetic and Dobry [39] for cohesionless soils. The curves adopted for models M1 and M2 are almost coincident and provide a close match with literature data for non-plastic soils.

\subsection{Validation of the model: 1D analyses}

The performance of the two constitutive models during dynamic loading, as well as the introduction of a small Rayleigh damping to overcome the inability of the models to dissipate energy at small strains, was verified through 1D wave propagation analyses, in which the horizontal acceleration time histories recorded at the base of the model container during test T4 (accelerometer A13) were applied at the bottom of a 1D soil column. The horizontal accelerations computed from 1D analyses were compared with those recorded in the centrifuge model by transducers A14 and A9, which are considered representative of free-field soil conditions.

Figure 4 shows a comparison between numerical and experimental accelerations (A9) during earthquake EQ1. The constitutive model M1 was adopted for the soil, using both $a_{1}=0.50$ (Fig. 4a, b) and $a_{1}=0.85$ (Fig. 4c, d), while three different values of the Rayleigh damping were used, that is, $D=0,2,4 \%$ and $f=f_{\text {inp, }}$, where $D$ is the minimum value of the viscous damping and $f$ is the frequency at which the minimum is attained. It is evident that the particular choice of the viscous damping does not affect the numerical results up to about $180 \mathrm{~Hz}$, where most part of the energy is contained in the input signal. On the other hand, higher frequencies are over-amplified in the numerical model if no Rayleigh damping is provided, resulting in unrealistic oscillations of accelerations within the soil mass. This fact, which does not depend on the constitutive assumptions of $a_{1}$, that is on the shear modulus degradation with increasing strain level, is clearly due to the inability of hysteretic constitutive soil models to provide sufficient damping at small strains $[14,22]$. Based on these observations, a $4 \%$ Rayleigh damping was used in the $2 \mathrm{D}$ analyses, with both soil models M1 and M2. 

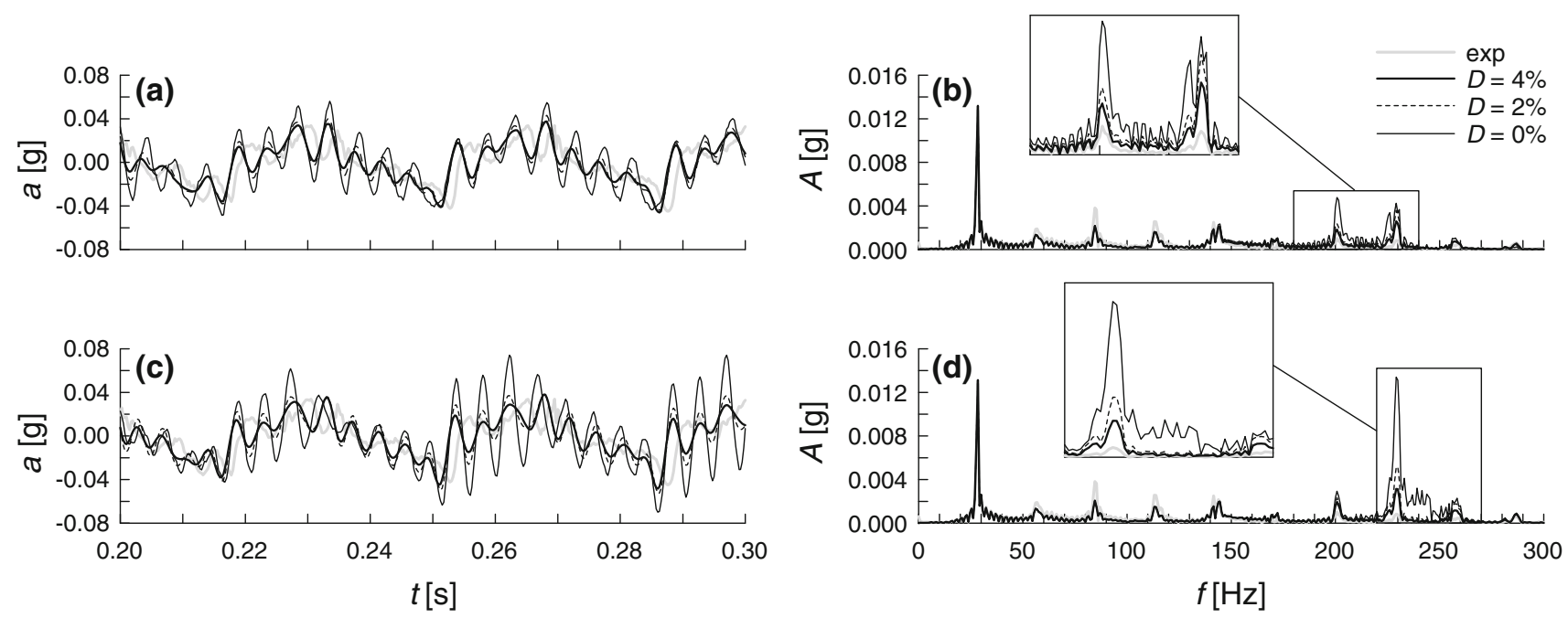

Fig. 4 Test T4, earthquake EQ1 (accelerometer A9): 1D wave propagation analyses with soil model M1: a, b $a_{1}=0.50$ and $\mathbf{c}, \mathbf{d} a_{1}=0.85$. Comparison between experimental data and numerical results obtained with different values of the Rayleigh damping
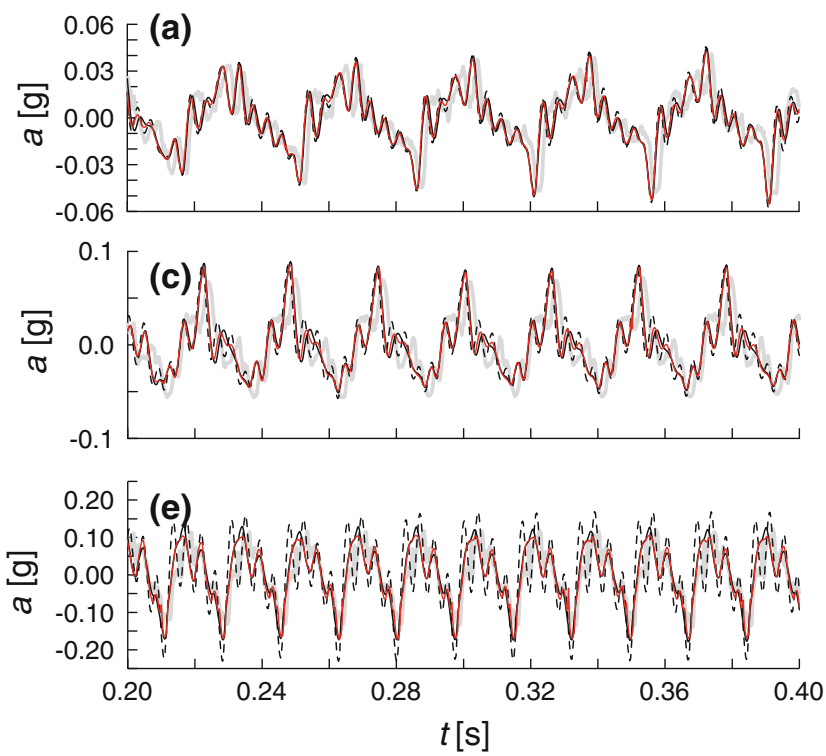
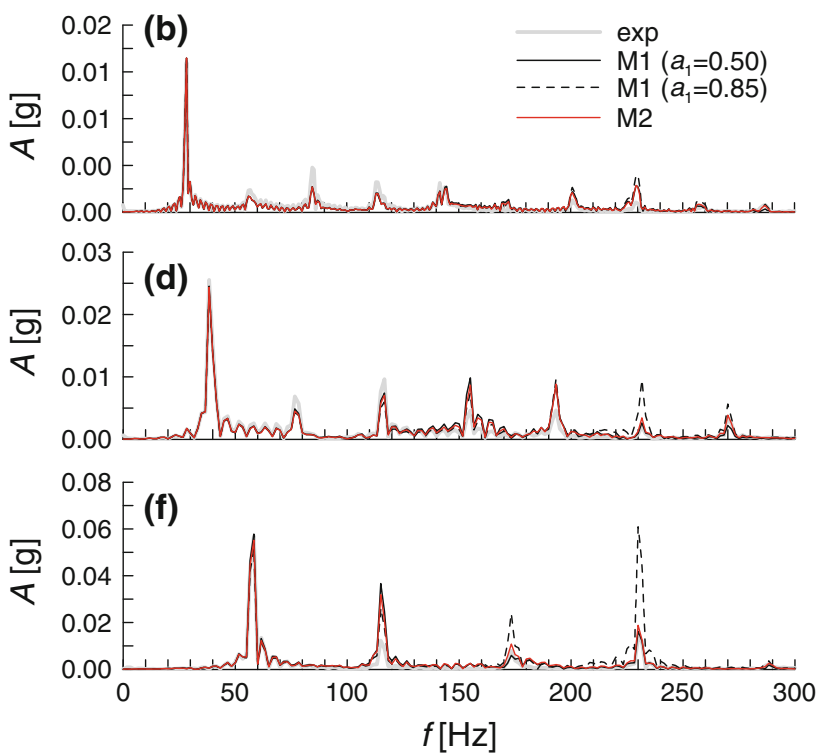

Fig. 5 Test T4: 1D wave propagation analyses for EQ2 (accelerometer A9: a, b), EQ4 (accelerometer A14: c, d) and EQ1 (accelerometer A9: e, f). Comparison between experimental data and numerical results

Figure 5 shows a comparison between numerical and experimental accelerations during earthquakes EQ2 (a, b), EQ4 (c, d) and EQ1 (e, f). Numerical analyses with soil model M1 were carried out adopting two different degradation curves for the shear modulus, that is $a_{1}=0.50$ and $a_{1}=0.85$. The shape of the $G / G_{0}$ curve has a negligible influence on the numerical results of EQ1, during which small shear strains are induced into the soil column. On the other hand, the choice of $a_{1}$ clearly affects the numerical predictions for both EQ2 and EQ4, as high frequency components are amplified unrealistically when $a_{1}$ is set equal to 0.85 (Fig. 5d, f). This observation, which is even more evident at larger accelerations (see, e.g. [10]), results from the fact that the $G / G_{0}$ curve derived from the best-fit of the laboratory data reported by Visone and Santucci de Magistris [38] does not describe adequately the nonlinear behaviour exhibited by the soil with increasing strain. Finally, numerical analyses carried out with models M1 and M2 provide almost the same results, and both models describe adequately the shear wave propagation through the soil layer.

\section{Numerical model}

The two-dimensional plane strain finite difference analyses were carried out at the model scale, by simulating both the 


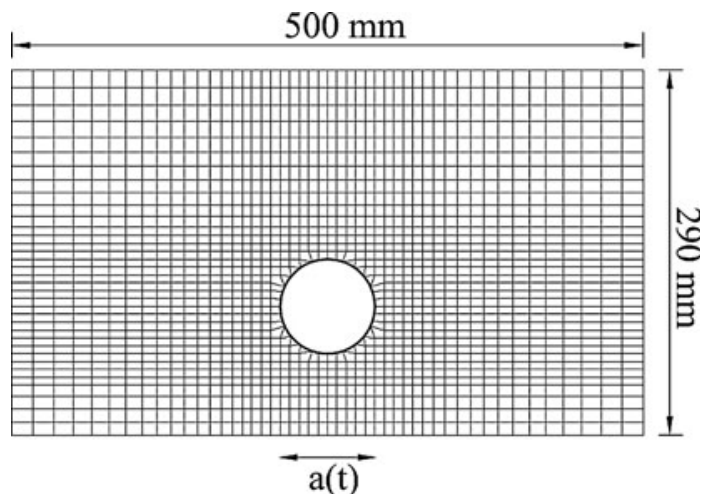

Fig. 6 Mesh used in the 2D numerical analyses (model scale)

static swing-up stage, during which the centrifugal acceleration into the model is increased from 1 to $80 \mathrm{~g}$, and the subsequent dynamic stages. Figure 6 shows the mesh adopted for the two tests, with a total of 1,610 elements and a minimum size of $6 \mathrm{~mm}$ near the tunnel. A coarser mesh was used for the analyses carried out with the advanced constitutive model M1, in order to reduce the computational time. In both cases, however, the refinement of the grid was chosen in order not to influence the numerical results during both the static and the dynamic stages. To this end, the element size $\Delta l$ always guarantees an accurate wave transmission through the model, that is $\Delta l \leq \lambda / 8$ [24], where $\lambda$ is the wavelength associated with the highest frequency of the input signals.

The structural elements were modelled as elastic isotropic beams attached directly to the grid nodes (no-slip condition). However, in order to study the influence of the contact condition between the lining and the soil on the computed internal forces, a further analysis was carried out, for the sole test T3 and soil model M2, in which elastic-perfectly plastic interfaces were adopted. A friction angle $\delta=12^{\circ}$ was used, which is a realistic value for the contact friction angle between aluminium alloy plates and LB Sand [26], while the normal and shear stiffness were set equal to $k_{\mathrm{s}}=k_{\mathrm{n}}=4 \times 10^{7} \mathrm{kN} / \mathrm{m}^{2} / \mathrm{m}$, which is about ten times the equivalent stiffness of the stiffest neighbouring zone [20].

The initial stress state was prescribed in terms of the earth pressure coefficient at rest $\sigma_{\mathrm{h}}^{\prime} / \sigma_{\mathrm{v}}^{\prime}=K_{0}\left(=1-\sin \phi_{\mathrm{cv}}\right)$, while an initial void ratio $e_{0}=0.71\left(D_{\mathrm{r}}=75 \%\right)$ and $e_{0}=0.85$ $\left(D_{\mathrm{r}}=40 \%\right)$ was adopted for test T3 and T4, respectively. It is worth observing that while in model M1, the relative density governs both the small strain shear stiffness and the contractant-dilatant behaviour of the soil, through the state parameter $\psi$; in model M2, the initial void ratio is taken into account for the sole definition of $G_{0}$ via Eq. (3).

During the swing-up stage, standard boundary conditions were applied to the model, i.e. zero horizontal displacements along the lateral boundaries and fixed nodes at the base of the grid, and the gravitational acceleration into the model was increased gradually from 1 to $80 \mathrm{~g}$ in successive steps.

After the swing-up stage, static constraints were removed from the boundaries. The input acceleration time histories (A13) were applied to the bottom nodes of the grid, together with a zero velocity condition in the vertical direction. Standard periodic constraints [46] were applied to the nodes on the lateral boundaries of the grid, i.e. they were tied to one another in order to enforce the same displacements in both the vertical and horizontal directions.

Time increments of $\Delta t=1.0 \times 10^{-7} \mathrm{~s}$ (model M1) and $\Delta t=5.0 \times 10^{-8} \mathrm{~s}$ (model M2) were adopted in the analyses in order to guarantee the stability of the explicit time integration scheme, the difference arising from the fact that a different mesh refinement was chosen for the two models.

\section{Numerical results}

Figure 7 shows the distribution of axial forces, $N$, and bending moments, $M$, in the tunnel at the end of the swingup stage, for test T3 (a, b) and T4 (c, d), respectively. Significant discrepancies can be observed between experimental data and numerical predictions, especially in terms of hoop forces, which are up to one order of magnitude larger than the experimental values. On the other hand, the results of the numerical analyses carried out using constitutive models M1 and M2 are almost the same, with a maximum difference of about $15 \%$ in terms of maximum hoop force. Moreover, as shown in Fig. 7a, the interface assumption between the lining and the soil does not affect substantially the numerical (static) predictions, at least for the contact friction angle considered in this work.

As far as the axial forces are concerned, the hoop force in the lining has been computed also assuming a uniform distribution of contact stresses as $N=\sigma_{0} R$, where $\sigma_{0}=80 g \times \rho z^{*}\left(1+K_{0}\right) / 2$ is the mean pressure acting on the lining, $z^{*}=187.5 \mathrm{~mm}$ is the depth of the tunnel axis and $K_{0}=1-\sin \phi$ is the earth pressure coefficient at rest. The values of $N=6.4 \mathrm{~N} / \mathrm{mm}$ and $N=5.9 \mathrm{~N} / \mathrm{mm}$ have been obtained for test $\mathrm{T} 3$ and $\mathrm{T} 4$, respectively, which are in close agreement with the mean values of $N$ provided by the numerical analyses. Note that the theoretical value of $N=\sigma_{0} R$ corresponds also to the mean value of the axial force that would be induced in the lining by a nonuniform distribution of contact stresses, as in the case of a tunnel under a geostatic stress field, and hence, it is representative of the mean value of the hoop force that would be expected in the tunnel for the two centrifuge tests at hand. On the contrary, the maximum bending moment in the lining depends strongly on the particular distribution of stresses 

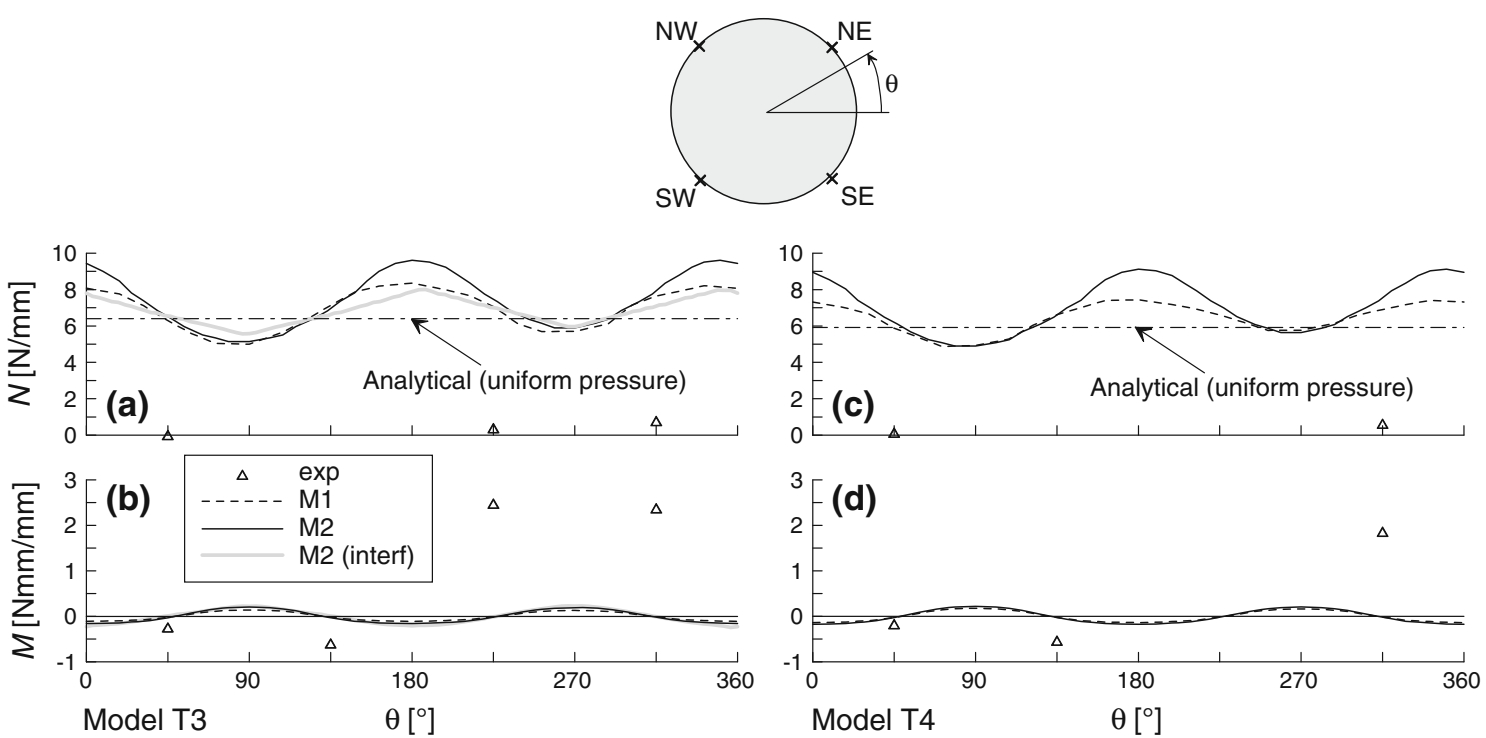

Fig. 7 Distribution of bending moments and hoop forces in the lining after the swing-up stage for: $\mathbf{a}, \mathbf{b}$ model T3 and $\mathbf{c}, \mathbf{d}$ model T4

acting on the tunnel (see, e.g. [6]). Following these observations, it is believed that the discrepancies observed in terms of bending moment could be related to some differences between the numerical and the experimental models, such as local nonuniformities of the sand in the centrifuge tests, while the very large differences obtained in terms of axial forces could be hardly attributed to the particular choice of the constitutive model for the soil or of the contact condition between the tunnel and the soil, and could be due instead to some error in the interpretation of the strain gauges measurements.

Figure 8 shows a comparison between computed and recorded acceleration time histories along the tunnel vertical (accelerometers A4, A6 and A8) during earthquakes (a) EQ2 and (b) EQ4 of test T3 and (c) earthquake EQ1 of test T4. As already observed in 1D analyses, numerical results are in quite good agreement with the experimental data, independently on the nominal frequency or amplitude of the applied signal, and no appreciable differences can be observed using the two different soil models M1 and M2.

A further comparison between predicted and measured accelerations is presented in Fig. 9, which shows the profiles of maximum accelerations along the free-field vertical (accelerometer A5, A7, A14 and A9) for the four earthquakes applied in tests T3 (a) and T4 (b). In both tests, measured accelerations show a slight de-amplification at the tunnel depth and a successive amplification close to the soil surface, this trend being less pronounced in the numerical analyses. Moreover, while the numerical predictions for test T3 are in good agreement with the centrifuge data, maximum accelerations at shallow depths are always overestimated in the numerical simulation of test $\mathrm{T} 4$ on loose sand.
Figure 10 shows the profile of maximum shear strains computed numerically along the free-field vertical during the four earthquakes applied in tests T3 (a) and T4 (b). Again, the two constitutive models M1 and M2 provide approximately the same description of the soil behaviour in all the applied earthquakes. Maximum deformations at the tunnel depth range from $0.01 \%$ (EQ1) to $0.1 \%$ in the stronger earthquake EQ4. The minimum wavelength associated with the applied accelerations can be computed as $\lambda_{\text {min }}=V_{\mathrm{S} \text {, min }} / f_{\text {max }}$, where $f_{\text {max }} \cong 320 \mathrm{~Hz}$ is the highest frequency of the input signals and $V_{\mathrm{S}, \min } \cong 160 \mathrm{~m} / \mathrm{s}$ is the minimum shear wave velocity at the tunnel depth, corresponding to a shear strain of about $0.1 \%\left(G / G_{0}=0.3\right)$. As $\lambda_{\text {min }} \cong 0.5 \mathrm{~m}$, and then, $D / \lambda_{\min } \ll 1$, it follows that the tunnel can be assumed to interact with a soil layer subjected to a uniform strain field.

Figure 11 shows the time histories of bending moment and hoop force in the lining, at angles of $\theta=135^{\circ}$ (NW) and $\theta=315^{\circ}(\mathrm{SE})$, respectively. Only the dynamic increments associated with earthquakes (a) EQ1 and (b) EQ4 of test T3 and (c) EQ1 of test T4 are reported, together with the corresponding values obtained introducing the interface elements between the tunnel and the surrounding soil. As far as the bending moments are concerned, the maximum (transient) values provided by the numerical analyses are in reasonable agreement with the experimental data, but the final (permanent) values are significantly underestimated. Once again, no significant differences are observed between models M1 and M2 and, as expected, the interface elements do not affect the numerical results. As already observed by Lanzano et al. [25], permanent increments in the internal forces in the lining are mainly due to sand densification. It is believed that the observed discrepancies 
(a) Test T3: earthquake EQ2

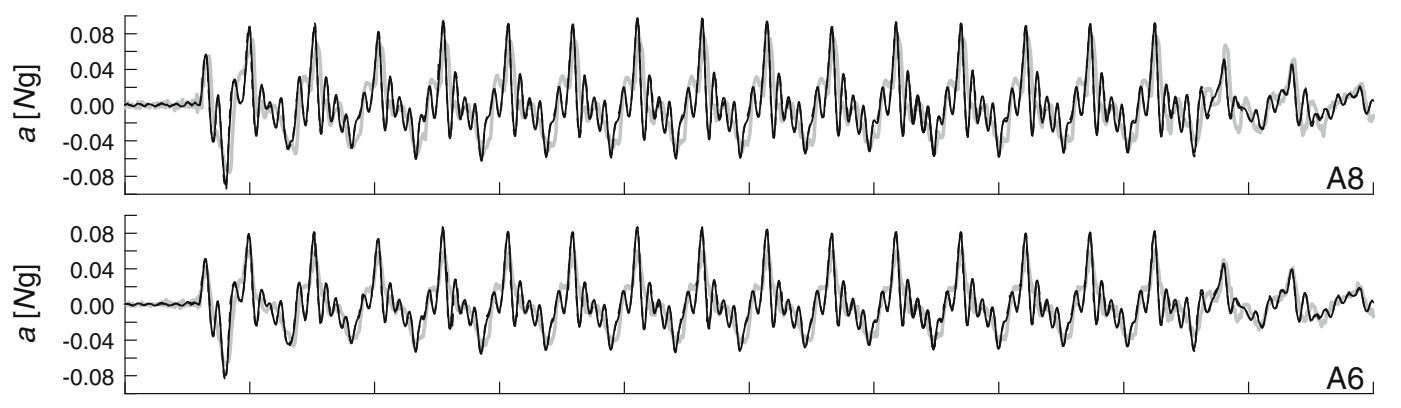

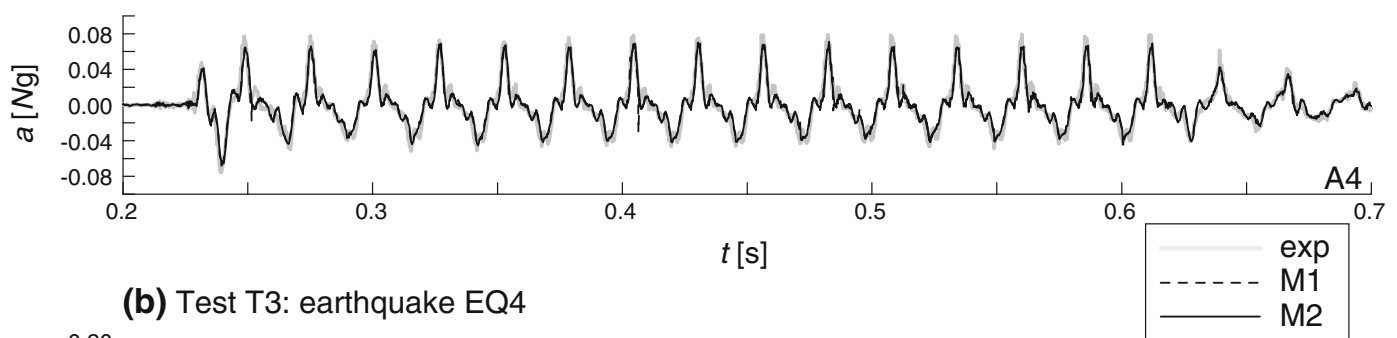

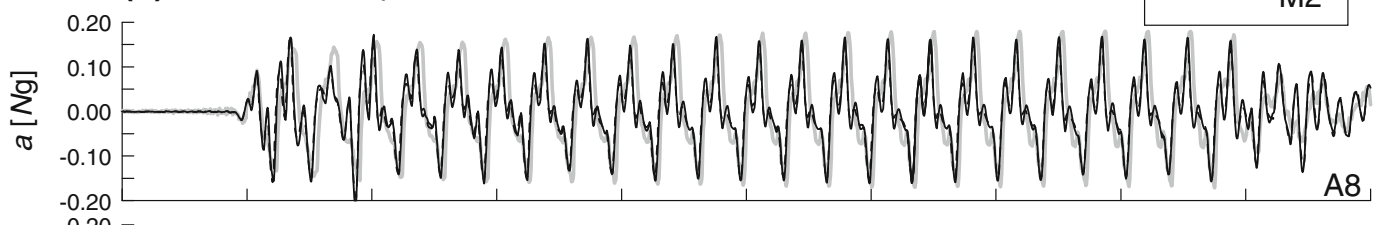

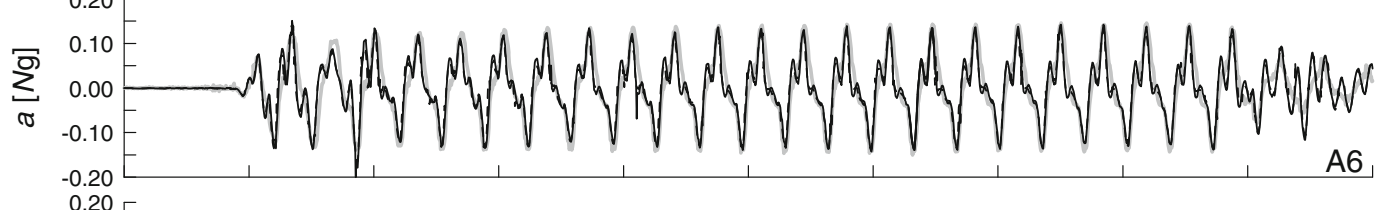

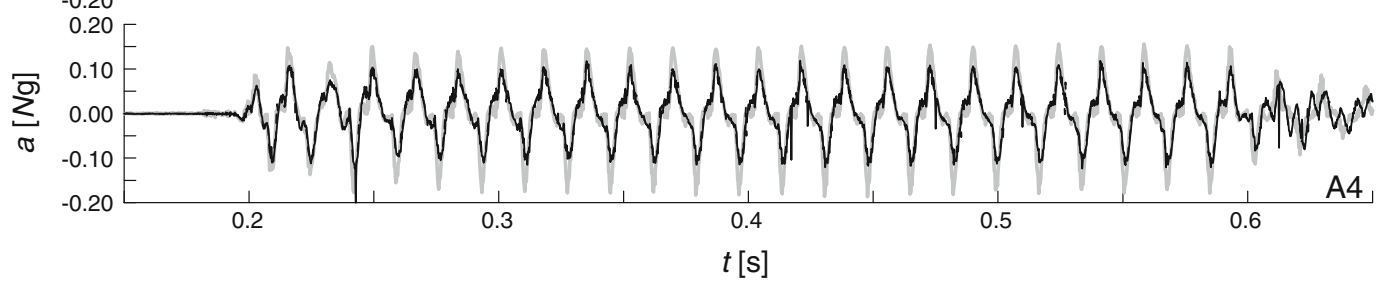

(c) Test T4: earthquake EQ1

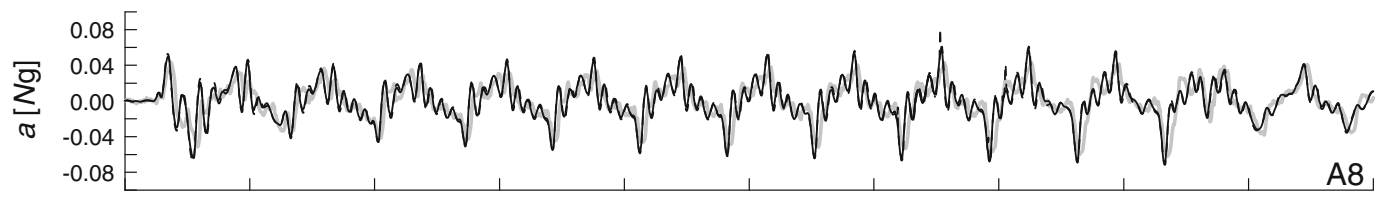

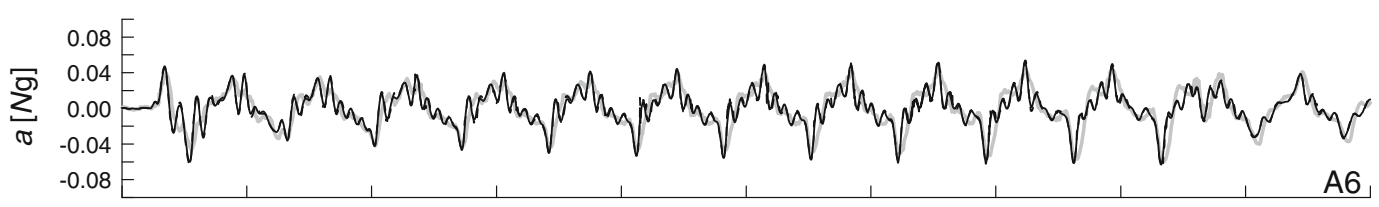

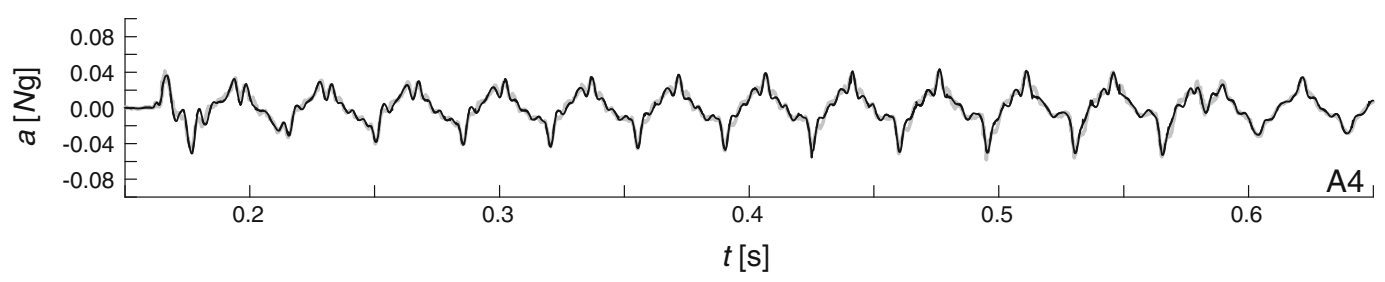

Fig. 8 Accelerations along the tunnel vertical (A4, A6 and A8) during earthquakes: a EQ2 and b EQ4 of test T3 and c EQ1 of test T4. Comparison between experimental data and numerical results 
maximum acceleration: $a_{\max } / \mathrm{N} \cdot \mathrm{g}$

(a)
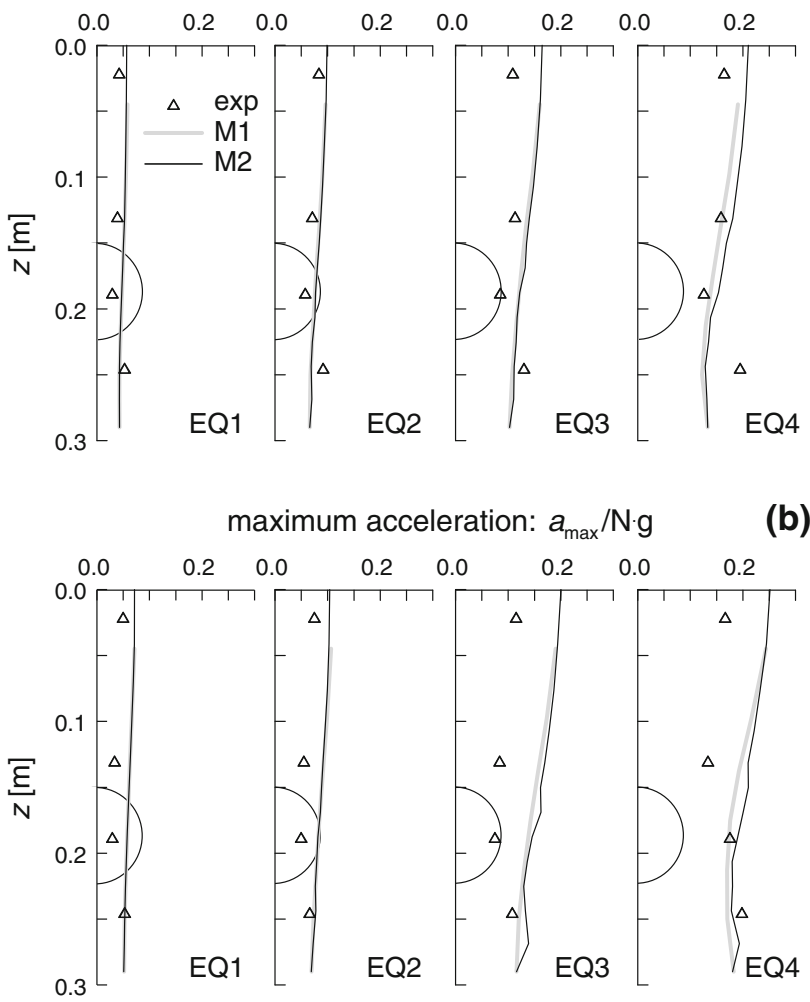

Fig. 9 Free-field vertical, distribution of maximum accelerations during the four earthquakes applied: a test $\mathrm{T} 3$ and $\mathbf{b}$ test $\mathrm{T} 4$. Comparison between experimental data and numerical results

in terms of permanent bending moments can be attributed to local nonuniformities of the sand close to the tunnel in the centrifuge models, which are not reproduced in the numerical analyses. As a matter of fact, during sand pouring, zones of smaller relative density could have been result close to the tunnel, due to the round shape of the lining.

A completely different scenario takes place in terms of hoop forces, where the numerical dynamic increments are more than one order of magnitude larger than the corresponding centrifuge values, irrespective of the contact condition between the lining and the soil. Moreover, in this case, the analyses carried out with soil model M1 provide larger values of the final (permanent) hoop force in the lining.

The same result is even more evident by inspection of Figs. 12 and 13, which show, for all the earthquakes of tests $\mathrm{T} 3$ and $\mathrm{T} 4$, respectively, the average values of the peak-to-peak amplitude of axial forces and bending moments, representative of the transient dynamic increments induced in the lining by the model excitation [25]. Accordingly, the figures also report the theoretical values obtained with the closed-form solutions for the no-slip condition (see "Appendix"), with reference to the maximum shear strain computed along the free-field vertical at
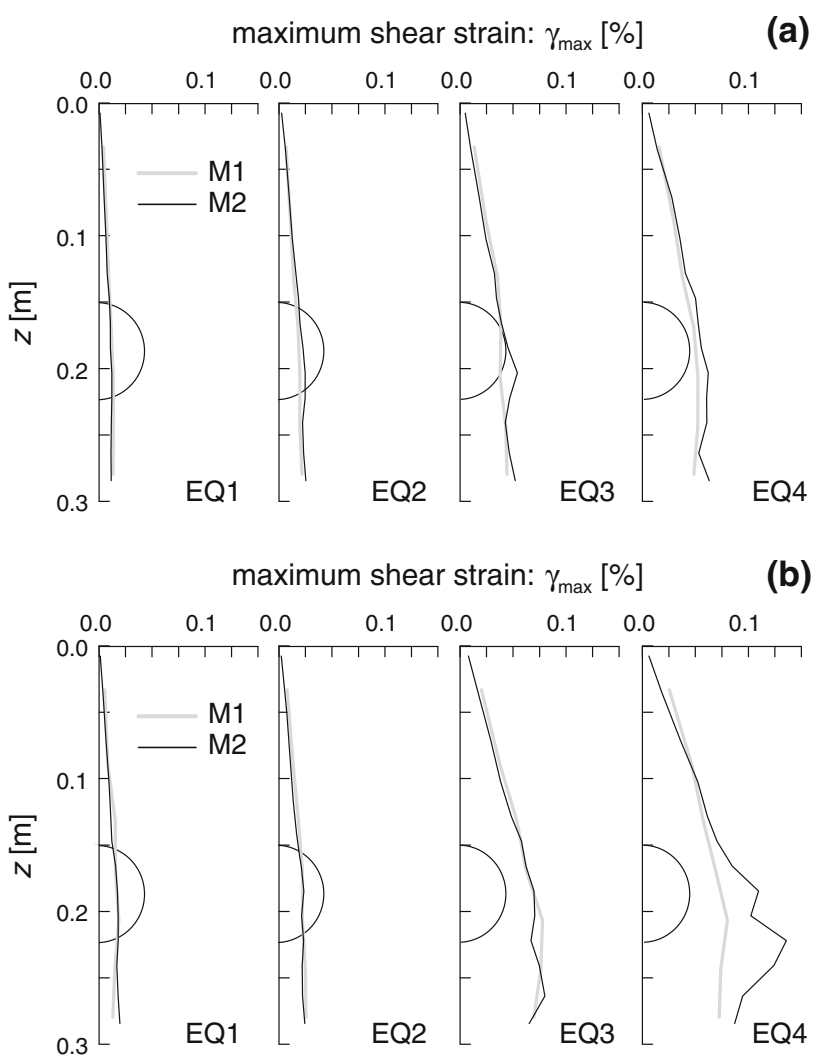

Fig. 10 Free-field vertical, distribution of maximum shear strain during the four earthquakes applied: a test T3 and b test T4

the tunnel depth, in the analyses carried out with model M2. Internal forces computed in the standard analyses (i.e. without interface elements) with the two constitutive models are quite similar to one another and in good agreement with the theoretical values, both in terms of bending moments and hoop forces. Moreover, as already shown in Fig. 11, numerical dynamic bending moments are similar to the experimental ones, at least to those measured at the polar angles of $\theta=135^{\circ}(\mathrm{NW})$ and $\theta=225^{\circ}(\mathrm{SW})$. On the other hand, experimental values of the dynamic increment in hoop forces are always significantly smaller than the numerical ones, even to those obtained with a more realistic representation of the contact condition between the tunnel and the soil. The same results were obtained by Kouretzis et al. [23] who observed that a better match with centrifuge data is achieved only when a zero friction condition at the sand-tube interface is assumed, as in Bilotta et al. [5].

Tables 4 and 5 report the maximum dynamic increments in bending moments and hoop forces in the lining, obtained from the two centrifuge tests and the corresponding numerical simulations, and computed with the close-form solutions assuming both the no-slip and the full-slip condition. As expected, the contact condition does not affect significantly the analytical predictions in terms of bending 


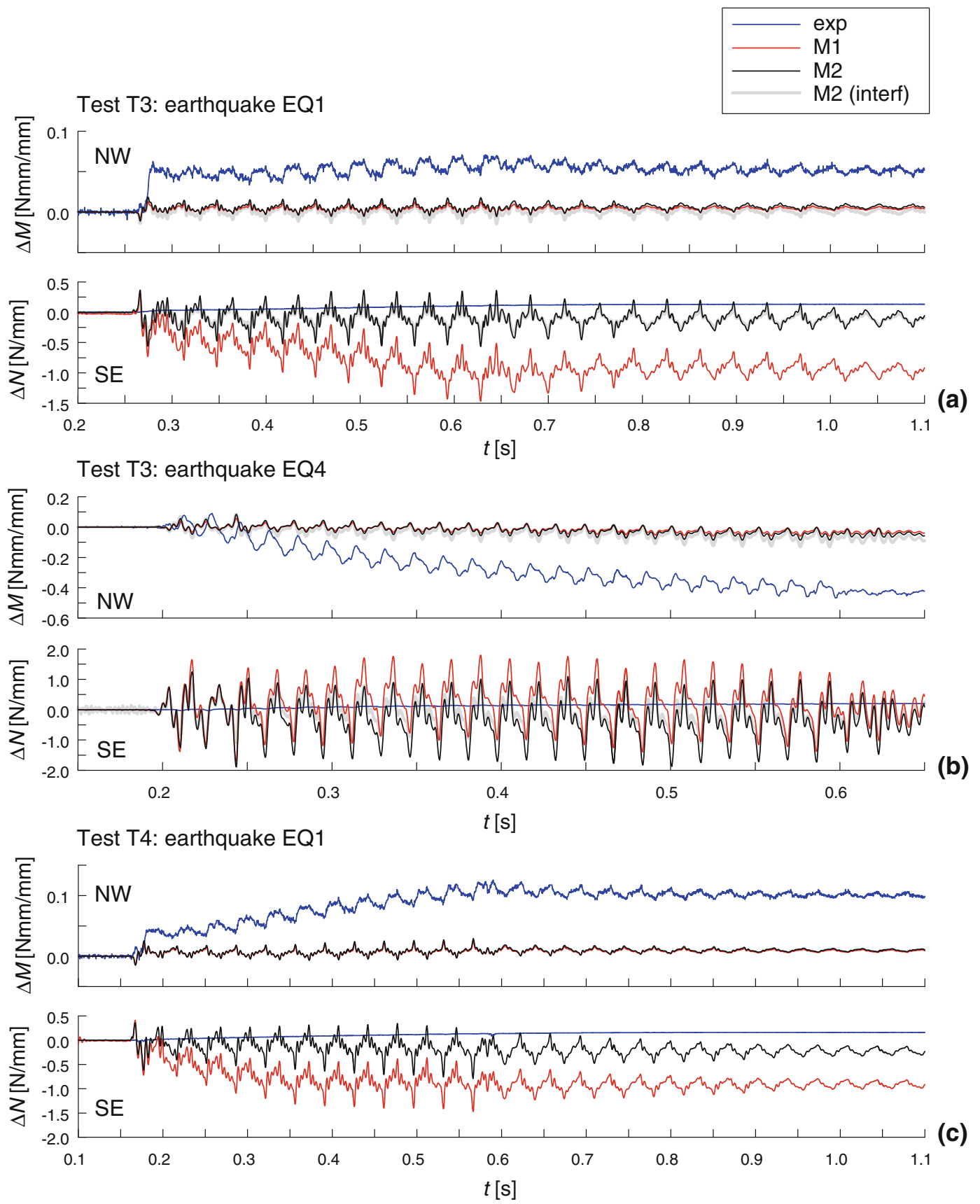

Fig. 11 Dynamic increment in bending moment (NW) and hoop force (SE) in the lining during earthquakes: a EQ1 and b EQ4 of test T3 and c EQ1 of test T4. Comparison between experimental data and numerical results

moments, as the values computed with the full-slip assumption are only slightly larger that those evaluated under the no-slip condition. On the contrary, the analytical values of the hoop force can vary up to three orders of magnitude, depending on the contact assumption. It is worth observing, however, that no agreement is achieved between centrifuge data and closed-form solutions even assuming zero friction between the tunnel and the soil. Moreover, this assumption seems to be quite unrealistic for the problem at hand. In fact, as stated by many authors (see, e.g. $[1,18]$ ), the full-slip condition at the interface is possible only under severe seismic loading conditions or for flexibility ratios $F<1$, as in the case of tunnels in very soft ground, while for the two centrifuge tests under examination, the flexibility ratio ranges between 800 and 2,300 , depending on the value of the shear modulus mobilised during each earthquake. Consistently with the results already discussed for the static condition, we believe that the discrepancies between numerical and centrifuge data in terms of hoop forces in the lining cannot 

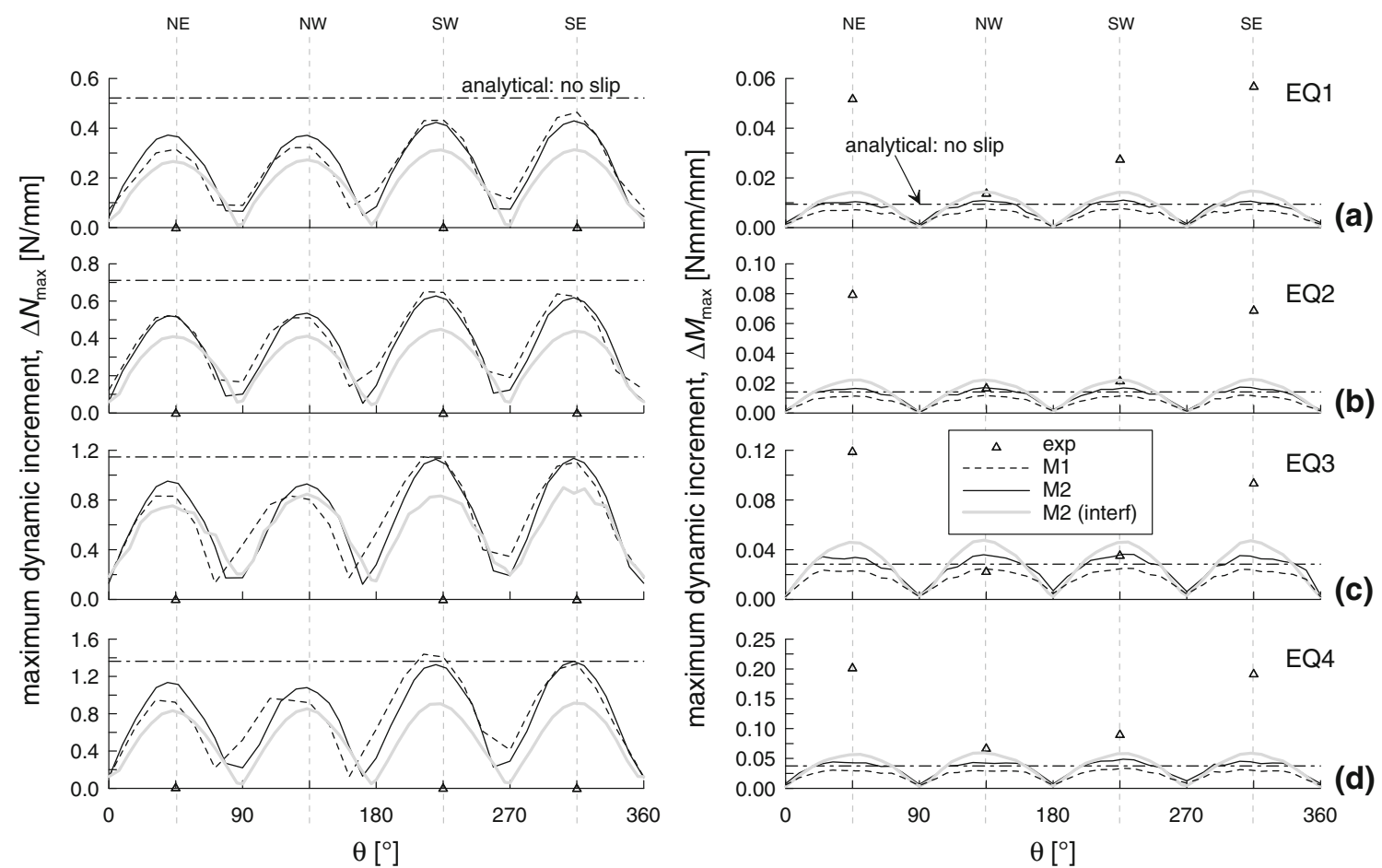

Fig. 12 Test T3. Maximum dynamic increment in bending moments and hoop forces in the lining during earthquakes: a EQ1, b EQ2, c EQ3 and d EQ4. Comparison between experimental data, numerical results and analytical solutions
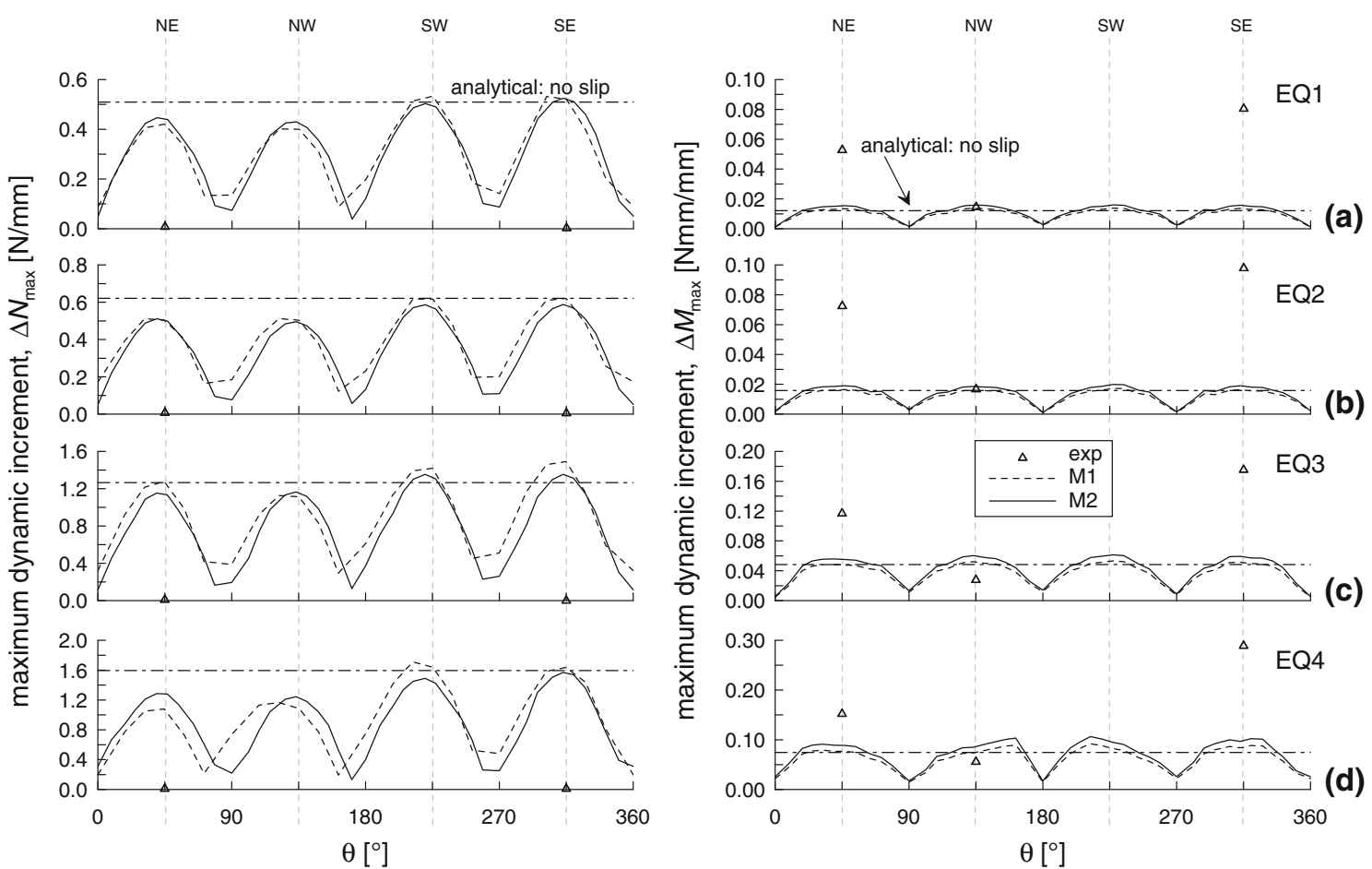

Fig. 13 Test T4. Dynamic increment in bending moments and hoop forces in the lining during earthquakes: a EQ1, b EQ2, c EQ3 and d EQ4. Comparison between experimental data, numerical results and analytical solutions 
Table 4 Maximum dynamic increment in bending moment in the liner: comparison between centrifuge data, numerical results and analytical predictions

\begin{tabular}{|c|c|c|c|c|c|c|c|}
\hline \multirow[t]{2}{*}{$\Delta M_{\max }(\mathrm{Nmm} / \mathrm{mm})$} & \multirow[t]{2}{*}{ Exp } & \multicolumn{4}{|c|}{ Numerical } & \multicolumn{2}{|c|}{ Analytical } \\
\hline & & $M 1$ & $M 2$ & $M 2$ (int) & $\gamma_{\max }(\%)^{\mathrm{a}}$ & Full-slip & No-slip \\
\hline \multicolumn{8}{|l|}{ Test T3 } \\
\hline EQ1 & 0.057 & 0.008 & 0.011 & 0.015 & 0.013 & 0.011 & 0.009 \\
\hline EQ2 & 0.080 & 0.012 & 0.017 & 0.023 & 0.019 & 0.017 & 0.014 \\
\hline EQ3 & 0.120 & 0.025 & 0.036 & 0.048 & 0.038 & 0.033 & 0.028 \\
\hline EQ4 & 0.203 & 0.033 & 0.049 & 0.059 & 0.050 & 0.044 & 0.038 \\
\hline \multicolumn{8}{|l|}{ Test T4 } \\
\hline EQ1 & 0.081 & 0.014 & 0.016 & - & 0.016 & 0.014 & 0.012 \\
\hline EQ2 & 0.099 & 0.017 & 0.020 & - & 0.021 & 0.019 & 0.016 \\
\hline EQ3 & 0.177 & 0.053 & 0.061 & - & 0.065 & 0.057 & 0.048 \\
\hline EQ4 & 0.292 & 0.092 & 0.106 & - & 0.101 & 0.089 & 0.075 \\
\hline
\end{tabular}

${ }^{a}$ Free-field shear strain at the tunnel depth (from 2D analyses with soil model M2)

Table 5 Maximum dynamic increment in hoop force in the liner: comparison between centrifuge data, numerical results and analytical predictions

\begin{tabular}{|c|c|c|c|c|c|c|c|}
\hline \multirow[t]{2}{*}{$\Delta N_{\max }(\mathrm{N} / \mathrm{mm})$} & \multirow[t]{2}{*}{ Exp } & \multicolumn{4}{|c|}{ Numerical } & \multicolumn{2}{|c|}{ Analytical } \\
\hline & & $M 1$ & $M 2$ & $M 2$ (int) & $\gamma_{\max }(\%)^{\mathrm{a}}$ & Full-slip & No-slip \\
\hline \multicolumn{8}{|l|}{ Test T3 } \\
\hline EQ1 & 0.0035 & 0.4640 & 0.4295 & 0.3133 & 0.013 & 0.0003 & 0.5213 \\
\hline EQ2 & 0.0033 & 0.6505 & 0.6280 & 0.4484 & 0.019 & 0.0004 & 0.7110 \\
\hline EQ3 & 0.0061 & 1.1474 & 1.1355 & 0.9004 & 0.038 & 0.0009 & 1.1463 \\
\hline EQ4 & 0.0148 & 1.4384 & 1.3625 & 0.9135 & 0.050 & 0.0012 & 1.3625 \\
\hline \multicolumn{8}{|l|}{ Test T4 } \\
\hline EQ1 & 0.0099 & 0.533 & 0.5249 & - & 0.016 & 0.0004 & 0.5092 \\
\hline EQ2 & 0.0141 & 0.621 & 0.5876 & - & 0.021 & 0.0005 & 0.6208 \\
\hline EQ3 & 0.0201 & 1.491 & 1.3544 & - & 0.065 & 0.0015 & 1.2646 \\
\hline EQ4 & 0.0305 & 1.711 & 1.5691 & - & 0.101 & 0.0024 & 1.5959 \\
\hline
\end{tabular}

${ }^{a}$ Free-field shear strain at the tunnel depth (from 2D analyses with soil model M2)

be attributed to an inaccurate reproduction of the experimental conditions in the numerical analyses.

\section{Discussion of results}

As shown in the previous sections, the two constitutive models, M1 and M2, provide almost the same predictions for the dynamic behaviour of the soil and, hence, for the overall soil-structure interaction problem analysed in this paper, the only significant difference being observed in terms of permanent internal forces in the lining. A further insight into the problem can be gained by inspection of Fig. 14, which shows the shear stress and strain time histories and the $\tau-\gamma$ cycles computed along the free-field vertical $(z=0.182 \mathrm{~m})$ during the earthquakes (a) EQ1 and (b) EQ2 of test T4.
The shear stress provided by the two models closely match. On the other hand, model M1 predicts a progressive accumulation of permanent shear strains, the transient component being instead quite similar to that obtained using model M2. This evidence results in the fact that the corresponding $\tau-\gamma$ cycles have almost the same slope, i.e. are characterised by the same value of the secant shear modulus, but the stationary cycles predicted by model M1 differ significantly from those obtained with model M2, this trend being more pronounced for stronger earthquakes.

These observations, which are intimately related to the ability of model M1 to reproduce sand fabric evolution during shearing [2, 30], allow to explain the observed difference in terms of permanent internal forces in the lining between the two models. It is worth noting, however, that the constant $N_{0}$, which governs the fabric evolution into the constitutive model M1, was chosen within the 
(a) earthquake EQ1
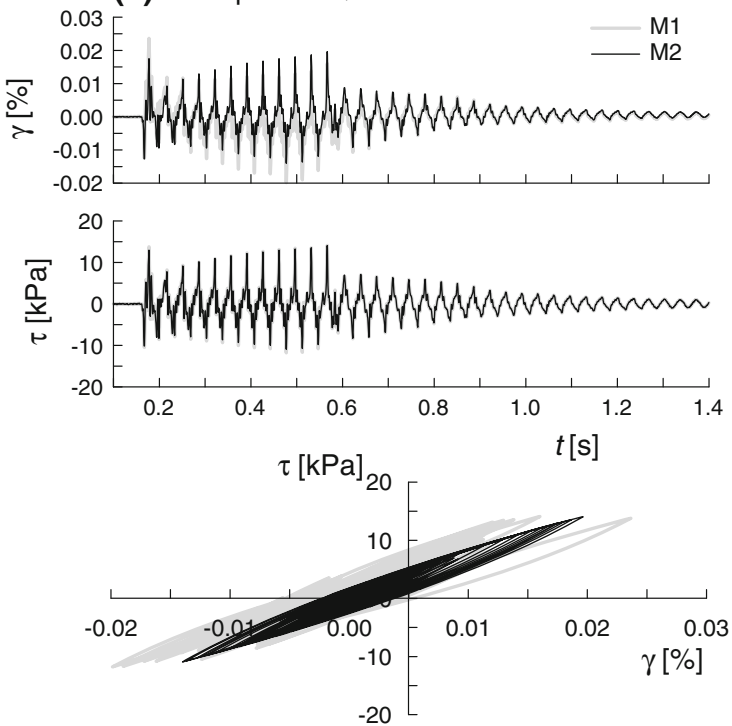

(b) earthquake $\mathrm{EQ} 2$
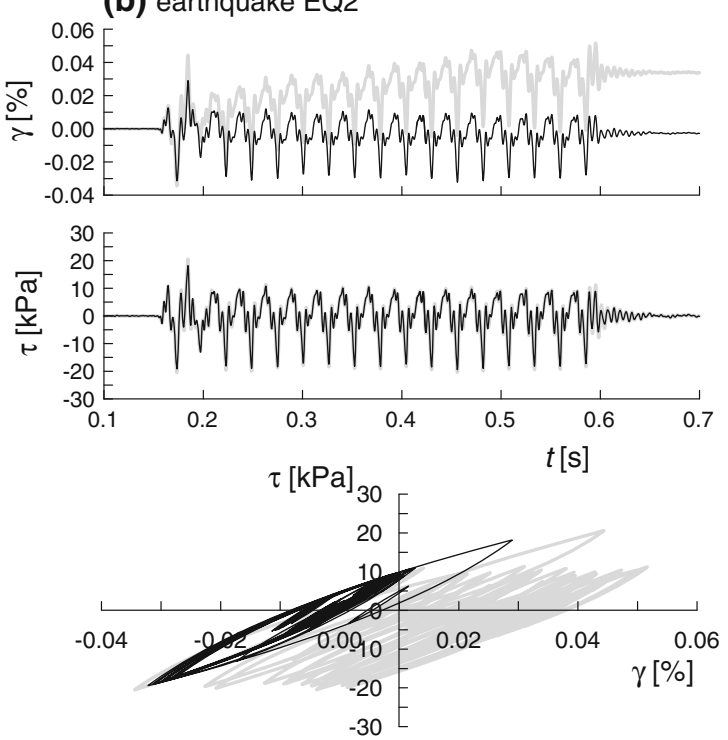

Fig. 14 Test T4, free-field vertical, $z=0.182 \mathrm{~m}$. Shear strain and shear stress time histories and $\tau-\gamma$ cycles during earthquake $\mathbf{a}$ EQ1 and $\mathbf{b}$ EQ2

typical range provided by Andrianopoulos et al. [2], as no experimental data were available for a proper calibration.

A final remark concerns the soil strength mobilisation during seismic loading. As shown in Fig. 14, the shear stresses induced into the soil are always smaller than the limiting value $\tau_{\lim }(=61 \mathrm{kPa}$ at $z=0.182 \mathrm{~m})$, this being true for all the earthquakes applied, thus suggesting that plasticity effects played a minor role in the numerical simulation of the two centrifuge tests. However, this is by no means a general conclusion as plasticity has been recognised to play a crucial role in the soil-tunnel interaction problem when strong earthquake is applied to the structure (see, e.g. [1]).

\section{Conclusions}

This paper has described the numerical simulation of two dynamic centrifuge tests on reduced scale models of shallow tunnels in dry sand, obtained using two different constitutive models, in order to compare their predictive capabilities and verify the effect of assumptions on the contact condition between the lining and the soil.

The values of bending moment and hoop force computed at the end of the swing-up stage with the two constitutive models are almost the same, with a maximum difference of about $15 \%$ in terms of maximum hoop force. The introduction of interfaces at the contact between the lining and the soil reduces the hoop forces by about $15 \%$. The agreement between numerical and experimental values is not very good, particularly in terms of hoop forces, which are up to one order of magnitude larger than the experimental values. However, the values of hoop force computed assuming a uniform distribution of contact stress equal to the mean pressure at the depth of the tunnel axis are close to the mean values provided by the numerical analyses.

For both tests T3 and T4, the computed and recorded accelerations are in good agreement with one another, independently on the nominal frequency or amplitude of the applied signal, and no appreciable differences can be observed using the two different soil models M1 and M2. In both tests, the numerical trend of de-amplification of acceleration at tunnel depth and successive amplification close to the soil surface is slightly less pronounced than measured. Moreover, while the numerical predictions for test T3 are in good agreement with the centrifuge data, maximum accelerations at shallow depths are always overestimated in the numerical simulation of test $\mathrm{T} 4$ on loose sand. Finally, for both tests T3 and T4, the two constitutive models provide approximately the same profile of maximum shear strains along the free-field vertical.

The computed maximum (transient) dynamic increments in bending moments are in good agreement with the experimental data, but the final (permanent) values are significantly underestimated. The predictions obtained using the two constitutive models are the same, and the introduction of interfaces at the contact between the soil and the lining does not affect the numerical results. On the other hand, the computed dynamic increments in hoop force are more than one order of magnitude larger than the corresponding experimental values, irrespective of the contact condition between the lining and the soil. The difference between the predictions of the final (permanent) 
hoop force obtained using the two constitutive models is more pronounced.

Based on a systematic comparison between experimental data, numerical predictions and theoretical results, both in static and dynamic conditions, it is believed that while the discrepancies observed in terms of bending moments could be related to some differences between the numerical and the experimental models, such as local nonuniformities of the sand in the centrifuge tests, the very large differences obtained in terms of axial forces could be due instead to some error in the interpretation of the strain gauges measurements.

The overall performance of the two constitutive models is very similar indicating that at least for dry sand, where shear-volumetric coupling is less relevant, the simple elastic-perfectly plastic model with nonlinear and hysteretic behaviour may provide an adequate representation of soil behaviour during the dynamic stages.

\section{Appendix}

The dynamic response of the tunnel, in the transverse direction, can be evaluated using a pseudostatic approach
$C=\frac{E_{\mathrm{s}}\left(1-v_{\mathrm{s}}^{2}\right) R}{E_{\mathrm{l}} t\left(1+v_{\mathrm{s}}\right)\left(1-2 v_{\mathrm{s}}\right)}$

Under full-slip conditions, the maximum increment in the hoop force $\left(\Delta N_{\max }\right)$ and the bending moment $\left(\Delta M_{\max }\right)$ in the lining is given by:

$\Delta N_{\max }= \pm \frac{1}{6} K_{1} \frac{E_{\mathrm{s}}}{\left(1+v_{\mathrm{s}}\right)} R \gamma_{\max }$

$\Delta M_{\max }= \pm \frac{1}{6} K_{1} \frac{E_{\mathrm{s}}}{\left(1+v_{\mathrm{s}}\right)} R^{2} \gamma_{\max }$

where:

$K_{1}=\frac{12\left(1-v_{\mathrm{s}}\right)}{2 F+5-6 v_{\mathrm{s}}}$

Under no-slip conditions, the maximum increment in the internal forces in the lining is given by:

$\Delta N_{\max }= \pm K_{2} \frac{E_{\mathrm{s}}}{2\left(1+v_{\mathrm{s}}\right)} R \gamma_{\max }$

$\Delta M_{\max }= \pm \frac{1}{2}\left(2-K_{2}-2 K_{3}\right) R^{2} \tau_{\max }$

where:
$K_{2}=1+\frac{F\left(1-2 v_{\mathrm{s}}\right)(1-C)-0.5 C\left(1-2 v_{\mathrm{s}}\right)+2}{F\left[\left(3-2 v_{\mathrm{s}}\right)+C\left(1-2 v_{\mathrm{s}}\right)\right]+0.5 C\left(5-6 v_{\mathrm{s}}\right)\left(1-2 v_{\mathrm{s}}\right)+\left(6-8 v_{\mathrm{s}}\right)}$
$K_{3}=\frac{F\left[1+C\left(1-2 v_{\mathrm{s}}\right)\right]-0.5 C\left(1-2 v_{\mathrm{s}}\right)-2}{F\left[\left(3-2 v_{\mathrm{s}}\right)+C\left(1-2 v_{\mathrm{s}}\right)\right]+0.5 C\left(5-6 v_{\mathrm{s}}\right)\left(1-2 v_{\mathrm{s}}\right)+\left(6-8 v_{\mathrm{s}}\right)}$ with the closed-form solutions provided by Wang [41], and extended recently by Kouretzis et al. [23], which compute the maximum increment in the internal forces in the lining under vertical propagating shear waves. The solutions refer to the two limit cases of zero friction (full-slip condition) and perfect bond (no-slip condition) between the tunnel and the surrounding soil, and are derived assuming: (1) plane strain conditions; (2) the soil is a homogeneous, elastic and isotropic medium; (3) the tunnel is circular; and (4) the ratio between the thickness of the lining and its diameter is small.

Two coefficients can be defined to quantify the relative stiffness between the soil and the tunnel, that is, the flexibility ratio, $F$, given by:

$F=\frac{E_{\mathrm{s}}\left(1-v_{1}^{2}\right) R^{3}}{6 E_{1} I\left(1+v_{\mathrm{s}}\right)}$

and the compressibility ratio, $C$, given by:
Equation (10) for the bending moment is derived from Kouretzis et al. [23], as no solution is provided by Wang [41] for the no-slip case.

\section{References}

1. Amorosi A, Boldini D (2009) Numerical modelling of the transverse dynamic behaviour of circular tunnels in clayey soils. Soil Dyn Earthq Eng 29:1059-1072

2. Andrianopoulos KI, Papadimitriou AG, Bouckovalas GD (2010) Bounding surface plasticity model for the seismic liquefaction analysis of geostructures. Soil Dyn Earthq Eng 30(10):895-911

3. Andrianopoulos KI, Papadimitriou AG, Bouckovalas GD (2010) Explicit integration of bounding surface model for analysis of earthquake soil liquefaction. Int J Numer Anal Method Geomech 34(15):1586-1614

4. Been K, Jefferies MG (1985) A state parameter for sands. Géotechnique 35(2):99-112

5. Bilotta E, Lanzano G, Russo G, Silvestri F, Madabhushi SPG (2009) Seismic analyses of shallow tunnels by dynamic 
centrifuge tests and finite elements. Proc 17th Int Conf Soil Mech Geotech Eng The academia and practice of geotechnical engineering 474-477. doi:10.3233/978-1-60750-031-5-474

6. Carranza-Torres C, Diederichs M (2009) Mechanical analysis of circular linings with particular reference to composite supports. For example, linings consisting of shotcrete and steel sets. Tunn Undergr Sp Technol 24:506-532

7. Cavallaro A, Maugeri M, Mazzarella R (2001) Static and dynamic properties of Leighton Buzzard sand from laboratory tests Proc $4^{\text {th }}$ Int Conf Recent Adv Geotech Earthquake Eng Soil Dyn. San Diego, California

8. Cilingir U, Madabhushi SPG (2011) A model study on the effects of input motion on the seismic behaviour of tunnels. Soil Dyn Earthq Eng 31:452-462

9. Cilingir U, Madabhushi SPG (2011) Effect of depth on seismic response of circular tunnels. Can Geotech J 48:117-127

10. Conti R (2010) Modellazione fisica e numerica del comportamento di opere di sostegno flessibili in condizioni sismiche. $\mathrm{PhD}$ thesis, Università degli Studi di Roma Tor Vergata, Roma, Italy (in italian)

11. Conti R, Viggiani GMB (2012) Evaluation of soil dynamic properties in centrifuge tests. $\mathrm{J}$ Geotech Geoenv Eng 138(7):850-859

12. Corigliano M, Scandella L, Lai CG, Paolucci R (2011) Seismic analysis of deep tunnels in near fault conditions: a case study in Southern Italy. Bull Earthq Eng 9:975-995

13. Dietz M, Muir Wood D (2007) Shaking table evaluation of dynamic soil properties Proc $4^{\text {th }}$ Int Conf Earthq Geotech Eng Thessaloniki, Greece, June 25-28, Paper No. 1196

14. Ghosh B, Madabhushi SPG (2003) A numerical investigation into effects of single and multiple frequency earthquake input motion. Soil Dyn Earthq Eng 23(8):691-704

15. Gomes RC (2013) Effect of stress disturbance induced by construction on the seismic response of shallow tunnels. Comput Geotech 49:338-351

16. Hardin BO, Drnevich VP (1972) Shear modulus and damping in soils: measurement and parameter effects. J Soil Mech Found Div 98(6):603-624

17. Hashash YMA, Hook JJ, Schmidt B, Yao JJ (2001) Seismic design and analysis of underground structures. Tunn Undergr Sp Technol 16:247-293

18. Hashash YMA, Park D, Yao JIC (2005) Ovaling deformations of circular tunnels under seismic loading, an update on seismic design and analysis of underground structures. Tunn Undergr Sp Technol 20:435-441

19. Hatzigeorgiou GD, Beskos DE (2010) Soil-structure interaction effects on seismic inelastic analysis of 3-D tunnels. Soil Dyn Earthq Eng 30(9):851-861

20. Itasca (2005) FLAC Fast Lagrangian Analysis of Continua v. 5.0. User's Manual

21. Kontoe S, Zdravkovic L, Potts DM, Menkiti CO (2008) Case study on seismic tunnel response. Can Geotech J 45(12):1743-1764

22. Kontoe S, Zdravkovic L, Potts DM, Menkiti CO (2011) On the relative merits of simple and advanced constitutive models in dynamic analysis of tunnels. Géotechnique 61(10):815-829

23. Kouretzis GP, Sloan SW, Carter JP (2013) Effect of interface friction on tunnel liner internal forces due to seismic S- and P-wave propagation. Soil Dyn Earthq Eng 46:41-51

24. Kuhlemeyer RL, Lysmer J (1973) Finite element method accuracy for wave propagation problems. J Soil Mech Found, ASCE 99(SM5):421-427
25. Lanzano G, Bilotta E, Russo G, Silvestri F, Madabhushi SPG (2012) Centrifuge modeling of seismic loading on tunnels in sand. Geotech Test J 35(6):1-16

26. Madabhushi SPG, Zeng X (2007) Simulating seismic response of cantilever retaining walls. J Geotech Geoenv Eng 133(5):539-549

27. Manzari MT, Dafalias YF (1997) The strength and dilatancy of sands. Géotechnique 47(2):255-272

28. Masing G (1926) Eigenspannungen und Verfertigung bim Messing Proc 2nd Int Congress on Applied Mechanics, Zurich

29. Papadimitriou AG, Bouckovalas GD (2002) Plasticity model for sand under small and large cyclic strains: a multiaxial formulation. Soil Dyn Earthq Eng 22(3):191-204

30. Papadimitriou AG, Bouckovalas GD, Dafalias YF (2001) Plasticity model for sand under small and large cyclic strains. J Geotech Geoenv Eng 127(11):973-983

31. Penzien J, Wu CL (1998) Stresses in linings of bored tunnels. Earthq Eng Struct Dyn 27:283-300

32. Penzien J (2000) Seismically induced racking of tunnel linings. Earthq Eng Struct Dyn 29(5):683-691

33. Ramberg W, Osgood WR (1943) Description of stress-strain curve by three parameters. Technical Note 902, National Advisory Committee for Aeronautics, Washington, D.C

34. Sedarat H, Kozak A, Hashash YMA, Shamsabadi A, Krimotat A (2009) Contact interface in seismic analysis of circular tunnels. Tunn Undergr Sp Technol 24:482-490

35. Seed HB, Idriss IM (1970) Soil moduli and damping factors for dynamic analysis Report No. EERC 70-10, University of California, Berkeley

36. St John CM, Zahrah TF (1987) Aseismic design of underground structures. Tunn Undergr Sp Technol 2(2):165-197

37. Visone C (2008) Performance-based design approach in seismic design of embedded retaining walls $\mathrm{PhD}$ thesis, Università degli Studi di Napoli Federico II, Naples, Italy

38. Visone C, Santucci de Magistris F (2009) Mechanical behaviour of the Leighton Buzzard sand $100=170$ under monotonic, cyclic and dynamic loading conditions Proc XIII Conf L'Ingegneria Sismica in Italia, ANIDIS, Bologna, Italy

39. Vucetic M, Dobry R (1991) Effect of soil plasticity on cyclic response. J Geotech Geoenv Eng 117(1):89-107

40. Vucetic M (1994) Cyclic threshold shear strains in soils. J Geotech Geoenv Eng 120(12):2208-2228

41. Wang JN (1993) Seismic design of tunnels: a state-of-the-art approach. Parsons, Brinckerhoff, New York Monograph 7

42. Yang D, Naesgaard E, Byrne PM, Adalier K, Abdoun T (2004) Numerical model verification and calibration of george massey tunnel using centrifuge models. Can Geotech J 41:921-942

43. Yashiro K, Kojima Y, Shimizu M (2007) Historical earthquake damage to tunnels in Japan and case studies of railway tunnels in the 2004 Niigataken-Chuetsu earthquake. Q Rep Railw Techn Res Inst 48(3):136-141

44. Yu H, Yuan Y, Qiao Z, Gu Z, Yang Z, Li X (2013) Seismic analysis of a long tunnel based on multi-scale method. Soil Dyn Earthq Eng 49:572-587

45. Zhang JM, Wang G (2012) Large post-liquefaction deformation of sand, part I: physical mechanism, constitutive description and numerical algorithm. Acta Geotech 7(2):69-113

46. Zienkiewicz OC, Bianic N, Shen FQ (1988) Earthquake input definition and the transmitting boundary condition Conf: Advances in computational non-linear mechanics, Editor: St. Doltnis I 109-138 